J. Clin. Chem. Clin. Biochem.

Vol. 20, 1982, pp. 221-225

\title{
Peroxidase aus Meerrettich: \\ Kinetische Studien und Optimierung der Aktivitätsbestimmung mit den Substraten $\mathrm{H}_{2} \mathrm{O}_{2}$ und $o$-Phenylendiamin
}

Von H. Gallati und H. Brodbeck

\section{Diagnostische Forschungs- und Entwicklungsabteilung der F. Hoffmann-La Roche \& Co. AG Basel (Schweiz)}

(Eingegangen am 9. Juni/26. Oktober 1981)

Zusammenfassung: Zur Optimierung der Aktivitätsbestimmung der Peroxidase, eines häufig benutzten Markierungsund Indikatorenzyms für Enzymimmunoassays, wird die Kinetik der katalytischen Reaktion in Abhängigkeit der $\mathrm{H}_{2} \mathrm{O}_{2}$ - und der $o$-Phenylendiamin-Konzentration bei verschiedenen pH-Werten untersucht. Zudem werden die Einflüsse der Puffersysteme, der Reaktionstemperatur und der Reaktionsdauer abgeklärt. Auf Grund dieser Ergebnisse wird ein Testverfahren vorgeșchlagen, das erlaubt, die Peroxidase bis zu einer Konzentration von $2 \mathrm{ng} / 1 \mathrm{zu}$ bestimmen.

\section{Horseradish peroxidase:}

Kinetic studies and optimization of the activity determination with the substrates $\mathrm{H}_{2} \mathrm{O}_{2}$ and o-phenylenediamine

Summary: To optimize the determination of the activity of peroxidase, an enzyme often used as a marker and indicator system in enzyme immunoassays, the kinetics of the catalytic reaction and its dependence on the $\mathrm{H}_{2} \mathrm{O}_{2}$ and $o$-phenylenediamine concentration at various $\mathrm{pH}$ values were investigated. In addition, the influence of buffer systems, the reaction temperature, and the reaction time were determined. Based on the results, a test procedure is proposed for the measurement of peroxidase down to concentrations of $2 \mathrm{ng} / \mathrm{l}$.

\section{Einfuihrung}

Die Nachweisgrenze bei den enz̃ym-immunolögischen Tests (ĖIA) wird wesentlich beeinflußt von der erreichbaren Empfindlichkeit der enzymatischen Indikatorreaktion. Mit der Peroxidase aus Meerrettich (Donor: hydrogenperoxide oxidoreductàse, EC 1.11.1.7) als häufigstem Merkièrungs- und Indikatòrenžym stehen neben einer hohen katalytischen Aktivität zugleich auch einfache und sehr empfindliche Nachweismethoden zur Verfugung. So kann z.B. mit den Substraten $\mathrm{H}_{2} \mathrm{O}_{2}$ und 2,2'-Azino=di-(3-ethyl-benzthiazolin-sulfonsäure-(6)) (ABTS) die Peroxidase bis zu einer Konzentration von $10 \mathrm{ng} / 1$ gemessen werden (1).

Die vorliegende Arbeit berichtet vom erfolgreichen Versuch, die Sensitivität der enzym-immunologischen Tests noch weiter zu steigern. Als Ergebnis kann ein einfaches Testverfahren vorgeschlagen werden, das erlaubt, die Peroxidase bis zu einer Konzentration von $2 \mathrm{ng} / \mathrm{l}$ zu bestimmen.

Zur Optimierung der Aktivitätsbestimmung der Peroxidase wurde vorerst die Kinetik der katalytischen Reaktion in Abhängigkeit von der $\mathrm{H}_{2} \mathrm{O}_{2}$ - und der $O$-Phenyl-
endiamin-Konzentration bei verschiedenen $\mathrm{pH}$-Werten untersucht. Zusätzlich wurde die Abhängigkeit der enzymatischen Substratumsetzung von der Reaktionstemperatur, von der Reaktionsdauer sowie von verschiedenen Puffersystemen abgeklärt. Dann wurde auch die Möglichkeit zum sofortigen und vollständigen Abstoppen der peroxidatischen Aktivität und zur Stabilisierung der entstandenen Farbintensität untersucht.

\section{Material und Methoden}

Peroxidase aus Meerrettich (Donor:hydrogen-peroxide oxidoreductase, EC 1.11.1.7) wurde in lyophilisierter Form von Boehringer Mannheim bezogen. Die Reinheitszahl dieses Präparates ist mit „,ca. 3,0" angegeben. Das Enzym wurde ohne weitere Reinigung eingesetzt.

Als o-Phenylendiamin wurde das 1,2-Phenylendiamin von Merck (Best.Nr. 7243) benützt. In einer speziellen Studie wurde dieses Substrat mit dem o-Phenylendiamin von Fluka (Best.Nr. 78410) sowie mit dem o-Phenylendiamin-dihydrochlorid von Sigma (P-3888) und von Fluka (Best.Nr. 78440) verglichen.

$\mathrm{H}_{2} \mathrm{O}_{2}$ wurde in einer $300 \mathrm{~g} / \mathrm{kg}$-Lösung von der Firma Lehner, Muttenz (Schweiz), bezogen.

Die übrigen Chemikalien waren von analytischer Reinheit. 
Für die Aktivitätsbestimmung der Peroxidase werden in einer Photometcr-Meßküvette von $10 \mathrm{~mm}$ Schichtdicke zu $1,0 \mathrm{ml}$ Testlösung $(0,1 \mathrm{~mol} / 1$ Natriumcitratpuffer vom $\mathrm{pH} 5,0$ mit $15 \mathrm{mmol} / \mathrm{lo}$-Phenylendiamin und $\left.5 \mathrm{mmol} / 1 \mathrm{H}_{2} \mathrm{O}_{2}\right) 0,05 \mathrm{ml}$ Peroxidaselösung (Enzymkonzentration in der Testlösung bis $100 \mathrm{ng} / \mathrm{l}$ ) zugemischt und bei $25^{\circ} \mathrm{C}$ die Absorptionszunahme während 30 Minuten bei der Wellenlänge $436 \mathrm{~nm}$ photometrisch gemessen. Abweichungen von diesem Testvorgehen werden bei den einzelnen Versuchen speziell erwähnt.

\section{Resultate und Diskussion}

Spektren des o-Phenylendiamin und des Kondensations: produkts 2,2'-Diamino-azobenzol

In Anlehnung an die Arbeit von $Y$. Omote et al. (2) über die Oxidation des $O$-Phenyldiamin zum 2,2'-Diamino-azobenzol in Anwesenheit von Natriumhydrid kann angenommen werden, daß auch unter der katalytischen Einwirkung der Peroxidase das $o$-Phenylendiamin zum 2,2'Diamino-azobenzol kondensiert wird.

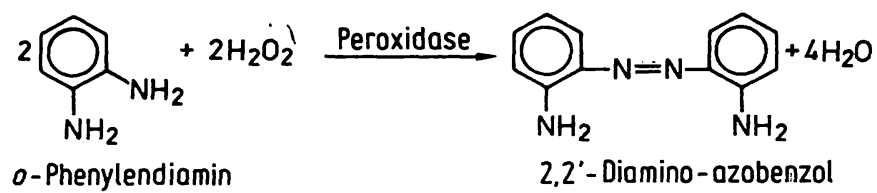

In Abbildung 1 sind die Spektren des $o$-Phenyldiamin sowie des 2,2'-Diamino-azobenzols aufgezeichnet. Der bei der peroxidatischen Oxidation gebildete Farbstoff zeigt ein Absorptionsmaximum bei $445 \mathrm{~nm}$. Bei Verwendung

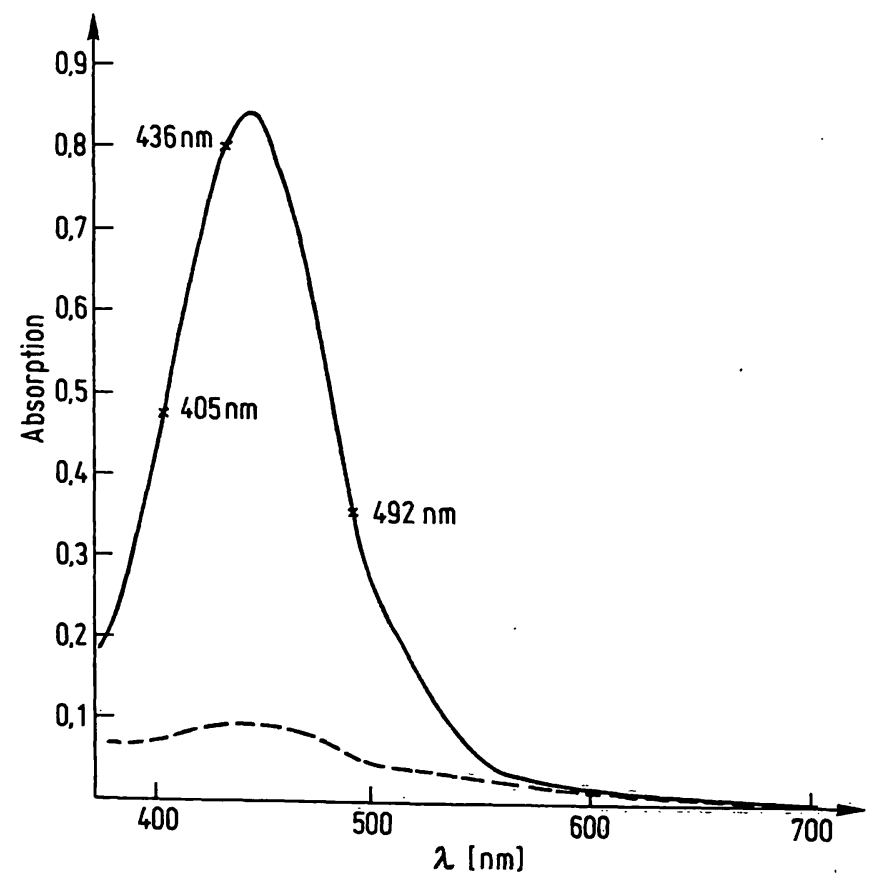

Abb: 1. Absorptionsspektren des o-Phenylendiamins und dés Kondensationsproduktes 2,2'-Diamino-azobenzol. Von $15 \mathrm{mmol} / \mathrm{l}$ o-Phenylendiamin vor ( -() und nach (-) der 60-minütigen katalytischen Einwirkung von $50 \mathrm{ng} / 1$ Peroxidase in Anwesenheit von $5 \mathrm{mmol} / 1 \mathrm{H}_{2} \mathrm{O}_{2}$ und $0,1 \mathrm{~mol} / 1$ Natriumcitratpuffer vom $\mathrm{pH} 5,0$ wurde das Spektrum im Wellenlängenbereich von $380 \mathrm{~nm}-\overline{700} \mathrm{~nm}$ aufgenommen. von gebräuchlichen Filtern für die photometrische Messung ist die Absorption bei $436 \mathrm{~nm}$ um $5 \%$, bei $405 \mathrm{~nm}$ um $44 \%$ und bei $492 \mathrm{~nm}$ um $56 \%$ geringer, was sich entsprechend auf die Empfindlichkeit der Aktivitätsbestimmung der Peroxidase auswirkt.

Für die folgenden Versuche wird die Aktivitätsbestimmung der Peroxidase bei der Wellenlänge $436 \mathrm{~nm}$ durchgeführt.

Bei den enzym-immunologischen Bestimmungen von Antigen oder Antikörpern nach der ELISA-Technik muß wegen der Heterogenität des Systems und wegen der geringen Reaktionsgeschwindigkeit die Aktivität der Peroxidase nach der „Zwei-Punkt-Methode“" bestimmt werden. Dabei ist es von praktischem Nutzen, wenn die Enzymreaktion nach Ablauf der festgesetżten Inkubationszeit abgestoppt und die gebildete Färbintensität innerhalb einer gewissen Zeit photometrisch gemessen werden kann. Bei Verwendung von $\mathrm{H}_{2} \mathrm{O}_{2}$ und $o$ Phenylendiamin als Substrat kann durch eine einfache pH-Senkung der Testlösung sowohl die katalytische Aktivität der Peroxidase sofort und vollständig abgestoppt wie auch die gebildete Farbintensität stabilisiert werden. Die Abbildung 2 zeigt die Spektren des 2,2'-Diaminoazobenzol bei verschiedenen $\mathrm{pH}$-Werten. Die Spektren bilden bei der Wellenlänge $452 \mathrm{~nm}$ einen isosbestischen . Punkt, was âls charakteristische Eigenschaft für einen

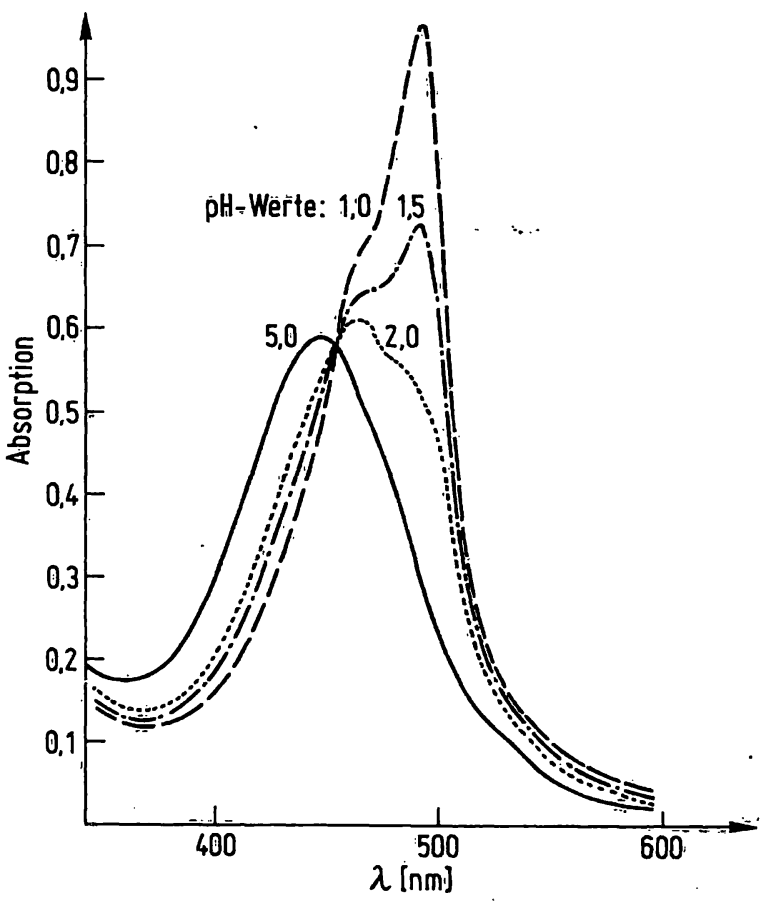

Abb. 2. Absorptionsspektren dès 2,2'-Diamiñò-azobenzols bei verschiedenen pH-Werten.

Nach einer 30-minütigen katalyțischen Einwirkung von $50 \mathrm{ng} / \mathrm{l}$ Peroxidase auf $15 \mathrm{mmol} / 1 \mathrm{o}$-P Phenylendiamin in Anwêsen̈heit von $5 \mathrm{mmol} / 1 \mathrm{H}_{2} \mathrm{O}_{2}$ und von $0,1 \mathrm{~mol} / 1$ Natriumcitratpuffer wurde durch Zugabe von konzentrierter Sālz̄säure dér pH-Wert der Testlösung vom pH 5,0 $(=)$ auf $2,0(\cdots \cdots)$, dann auf $1,5(-,-\cdot-\cdot)$ und auf $1,0(--)$ gesenkt. Von diesen Lösungen sind die Absorptionsspektren im Wellenlängenbereich von $350 \mathrm{~nm}$ bis $600 \mathrm{~nm}$ aufgenommen. 
pH-Indikator gilt. Durch die pH-Senkung nimmt die Farbintensität des 2,2'-Diamino-azobenzol wesentlich zu und zeigt ein Absorptionsmaximum bei der Wellenlänge $492 \mathrm{~nm}$.

Zum Abstoppen der Peroxidaseaktivität, zur Stabilisierung und zugleich Intensivierung der Farbintensität eignet sich jede Säure, welche den pH-Wert der Testlösung bis oder unter $\mathrm{pH} \mathrm{1,0}$ abzusenken vermag. Aus Praktikabilitätsgründen bevorzugen wir Salzsäure, auch wenn mit Schwefelsäure eine etwas bessere Stabilisierung der Farbintensität erreicht wird.

\section{Peroxidaseaktivität in Abhängigkeit vom pH-Wert und vom Puffersystem}

Bei einer gegebenen Substratkonzentration von $5 \mathrm{mmol} / \mathrm{I}$ $\mathrm{H}_{2} \mathrm{O}_{2}$ und $15 \mathrm{mmol} / \mathrm{l} o$-Phenylendiamin zeigt die katalytische Aktivität der Peroxidase beim pH 5,0 ein deutliches $\mathrm{pH}-$ Optimum, das von der Inkubationsdauer unabhängig ist (Abb. 3). Innerhalb des untersuchten $\mathrm{pH}$ Bereichs ist die Peroxidase im substratfreien Puffermilieu stabil. Das Enzym wird aber bei Anwesenheit von $\mathrm{H}_{2} \mathrm{O}_{2}$ langsam inaktiviert, indem die Peroxidase mit dem $\mathrm{H}_{2} \mathrm{O}_{2}$ einen stabilen und katalytisch inaktiven Komplex bildet (3-5).

In Natriumcitratpuffer $0,1 \mathrm{~mol} / 1$ wird unter sonst identischen Testbedingungen $\left(5 \mathrm{mmol} / 1 \mathrm{H}_{2} \mathrm{O}_{2}, 15 \mathrm{mmol} / 1\right.$ $o$-Phenylendiamin, pH 5,0 und $25^{\circ} \mathrm{C}$ ) die höchste Peroxidaseaktivität gemessen. Demgegenüber ist die peroxidatische Aktivität in Natriumacetat $0,1 \mathrm{~mol} / 1$ um $28 \%$, in Natriumtartrat $0,1 \mathrm{~mol} / 1 \mathrm{um} 30 \%$ und in Natriumphthalat $0,1 \mathrm{~mol} / 1 \mathrm{um} 40 \%$ geringer.

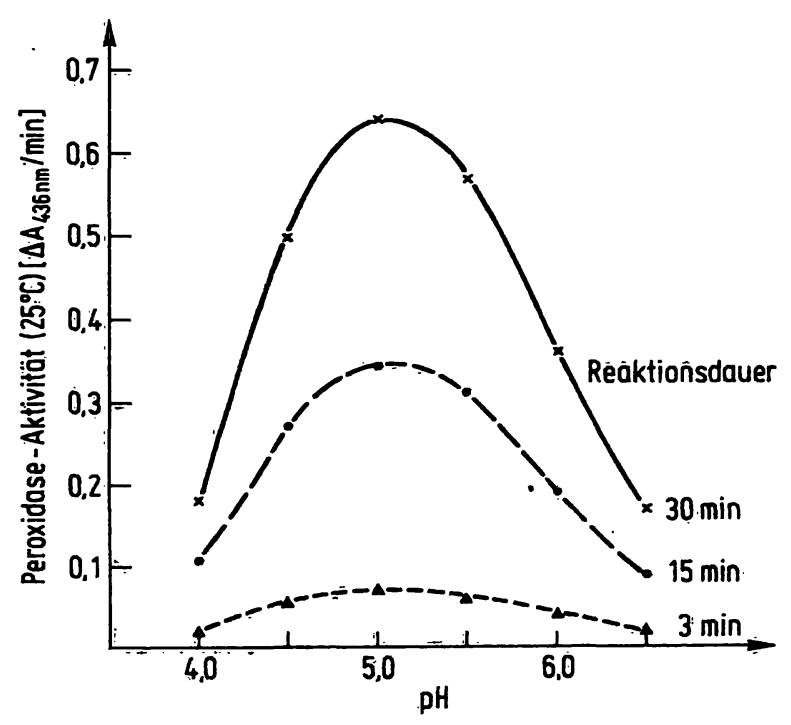

Abb. 3. pH-Aktivitätskurve der Peraxidase in Abhängigkeit der Inkubationsdauer.

Aktivitätsbestimmung von $80 \mathrm{ng} / 1$ Peroxidase in $0,1 \mathrm{~mol} / 1 \mathrm{Natriumcitrat} \mathrm{mit} 15 \mathrm{mmol} / 1 \mathrm{o}$-Phenylendiamin und $5 \mathrm{mmol} / \mathrm{l} \mathrm{H}_{2} \mathrm{O}_{2}$ bei den angegebenen $\mathrm{pH}$-Werten und der entsprechenden Inkubationsdauer. Reaktionstemperatur: $25^{\circ} \mathrm{C}$.
Natriumcitrat im Konzentrationsbereich von 50 bis $400 \mathrm{mmol} / 1$ hat keinen Einfluß auf die peroxidatische Aktivität. (Hingegen muß entsprechend der eingesetzten Pufferkapazität mehr oder dann konzentriertere Säure zum Abstoppen der enzymatischen Reaktion und zum Erreichen eines $\mathrm{pH}$-Wertes von 1,0 verwendet werden.)

\section{Peroxidaseaktivität in Abhängigkeit zur $\mathrm{H}_{2} \mathrm{O}_{2}$-Konzen- tration}

Die Peroxidaseaktivität wurde bei Anwesenheit von $15 \mathrm{mmol} / 1 o$-Phenylendiamin in Abhängigkeit der $\mathrm{H}_{2} \mathrm{O}_{2}$ Konzentration im pH-Bereich von 4,0 bis 6,0 untersucht und die Resultate nach der Darstellungsweise von Lineweaver-Burk in der Abbildung 4 zusammengefaßt.

Unter den gegebenen Testbedingungen ist die Affinität der Peroxidase zum $\mathrm{H}_{2} \mathrm{O}_{2}$ im $\mathrm{pH}$-Bereich von 4,0 bis 5,0 identisch und zeigt eine Michaelis-Konstante $K_{\mathrm{m}}=3,6 \mathrm{mmol} / 1$. Mit steigendem pH-Wert nimmt die $\mathrm{H}_{2} \mathrm{O}_{2}$-Affinität der Peroxidase stark zu: $\mathrm{pH}$ 5,5:

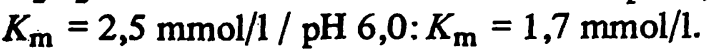

Die Abbildung 5 zeigt die Affinität der Peroxidase sowohl zum $\mathrm{H}_{2} \mathrm{O}_{2}$ wie auch zum $o$-Phenylendiamin $\left(\mathrm{p} K_{\mathrm{m}}=\log K_{\mathrm{m}}\right)$ in Korrelation zu den entsprechenden pH-Werten. Die beiden parallel verlaufenden Kurven żeigen beim pH 5,0 einen markanten Knick, wobei die Affinität der beiden Substrate zur Peroxidase mit steigendem pH-Wert gleichsinnig und stark zunimmt. Nach M. Dixon (6) bedeutet dieser Kurvenverlauf, daß die Ionisierung der für die Enzymaktivität entscheidenden Gruppe der Peroxidase zunimmt.

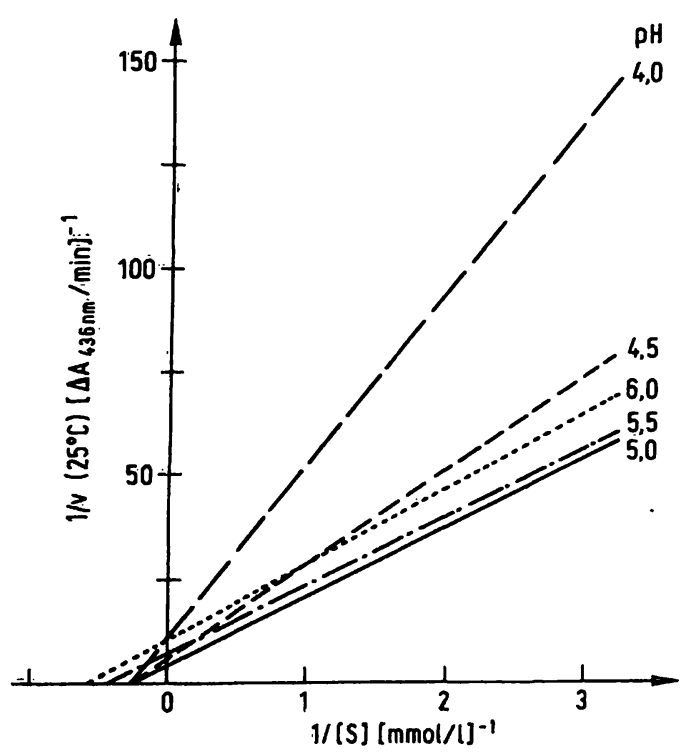

Abb. 4. Michaelis-Konstante der Peroxidase für $\mathrm{H}_{2} \mathrm{O}_{2}$ bei verschiedenen $\mathrm{pH}$-Werten.

Aktivitätsbestimmung von $100 \mathrm{ng} / 1$ Peroxidase in 0,1 mol/1 Natriumcitrat, $15 \mathrm{mmol} / 1 \mathrm{o}-\mathrm{Phenylendiamin} \mathrm{und}$ den entsprechenden $\mathrm{H}_{2} \mathrm{O}_{2}$-Konzentrationen bei den angegebenen pH-Werten. Reaktionstemperatur: $25^{\circ} \mathrm{C}$. Darstellung nach Lineweaver-Burk. 


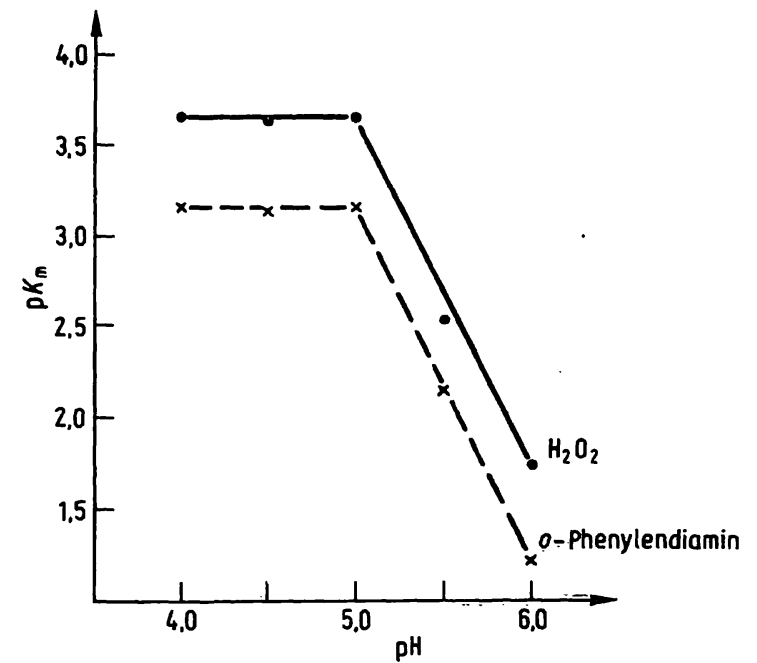

Abb. 5. Abhängigkeit der $\mathrm{p} K_{\mathrm{m}}$-Werte für $\mathrm{H}_{2} \mathrm{O}_{2}$ und o-Phenylendiamin vom $\mathrm{pH}$.

Die aus den Abbildungen 4 und 7 errechneten Michaelis: konstanten $K_{\mathrm{m}}$ werden als $\mathrm{p} K_{\mathrm{m}}\left(=-\log K_{\mathrm{m}}\right)$ in Abhängigkeit zum pH-Wert aufgetragen.

Zur Festsetzung der optimalen $\mathrm{H}_{2} \mathrm{O}_{2}$-Konzentration dürfen nicht nur die Resultate der Abbildung 4 berücksichtigt werden, die besagen, daß bei $\mathrm{pH}$ 5,0 die Peroxidaseaktivität mit steigender $\mathrm{H}_{2} \mathrm{O}_{2}$-Konzentration zunimmt. Denn, wie die Resultate der Abbildung 6 zeigen, nimmt mit höherem $\mathrm{H}_{2} \mathrm{O}_{2}$-Gehalt auch die Inaktivierungsgeschwindigkeit der Peroxidase zu. Zur Festsetzung der optimalen $\mathrm{H}_{2} \mathrm{O}_{2}$-Konzentration muß demnach auch die Inkubationsdauer der Peroxidase berücksichtigt werden. So wird bei einer Inkubationsdauer von 30 Minuten - bei sonst identischen Reak-

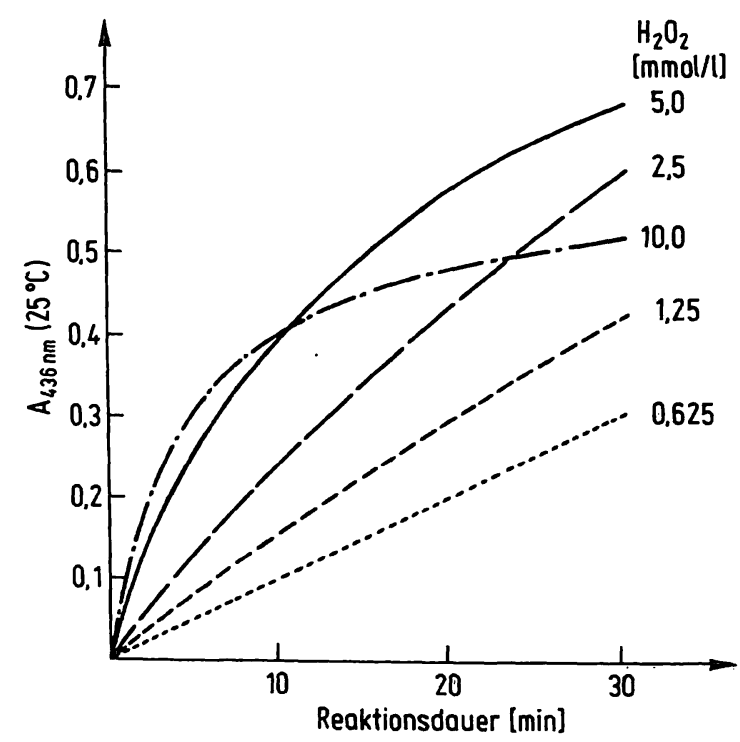

Abb. 6. Peroxidaseaktivität in Abhängigkeit zur $\mathrm{H}_{2} \mathrm{O}_{2}$ Konzentration und zur Reaktionsdauer.

$80 \mathrm{ng} / 1$ Peroxidase werden bei einer Reaktionstemperatur von $25^{\circ} \mathrm{C}$ in $0,1 \mathrm{~mol} / 1$ Natrium citrat vom pH 5,0 mit $15 \mathrm{mmol} / \mathrm{l} 0$-Phenylendiamin und verschiedenen $\mathrm{H}_{2} \mathrm{O}_{2}$-Konzentrationen inkubiert und die Absorption bei der Wellenlänge $436 \mathrm{~nm}$ während 30 Minuten verfolgt. tionsbedingungen - mit $5 \mathrm{mmol} / 1 \mathrm{H}_{2} \mathrm{O}_{2}$ die höchste Farbintensität erreicht. (Für 5 Minuten wären es $10 \mathrm{mmol} / 1 \mathrm{H}_{2} \mathrm{O}_{2}$ und für 60 Minuten $2,5 \mathrm{mmol} / 1$.)

Das Substrạt $o$-Phenylendiamin bietet der Peroxidase keinen Schutz vor der $\mathrm{H}_{2} \mathrm{O}_{2}$-Inaktivierung.

\section{Peroxidaseaktivität in Abhängigkeit zur o-Phenyl- endiamin-Konzentration}

In Abbildung-7 ist die Abhängigkeit der Peroxidaseaktivität vōn der $o$-Phenylendiamin-Konzentratiōn im untersuchten $\mathrm{pH}$-Bereich von 4,0 bis 6,0 nach dèr Darstellungsweise von Lineweaver-Burk zuisammengefaßit. Daraus kann die Affinität der Peroxidase zum $o$-Phenylendiamin als $K_{\mathrm{m}}$, wie auch die maximal möğliche Reaktionsgeschwindigkeit abgelesen werden. Die Korrelation der Affinität des $o$-Phenylendiamins zur Peroxidase im untersuchten pH-Bereich ist in Abbildün 5 dargestellt und öben kommentiert. Feštżuhälten ist, daß zur Substratsättigung der Peroxiddase wesentlich mehr $o$-Phenylendiamin eingesetzt wërden muß als $\mathrm{H}_{2} \mathrm{O}_{2}$. Die maximal möglichè Reaktionsgeschwindigkeit kann wegen der Substrathemmung hoher $o$-P̈heñylendiamin-Konzentrationen nicht erreicht werden. Eine optimale Peroxidaseaktivität wird beim pH 5,0 und in Anwesenheit von $5 \mathrm{mmol} / 1 \mathrm{H}_{2} \mathrm{O}_{2}$ mit $15 \mathrm{mmol} / 1$ o-Phenylendiamin erhalten.

Die Resultate der Tabelle 1 zeigen wesentliche Unter: schiede der Peroxidaseaktivität mit verschiedenen o-Phènylendiamin-Präparaten. Die höchste Aktivität wird mit dem o-Phenylendiamin von Merck gemeșsen,

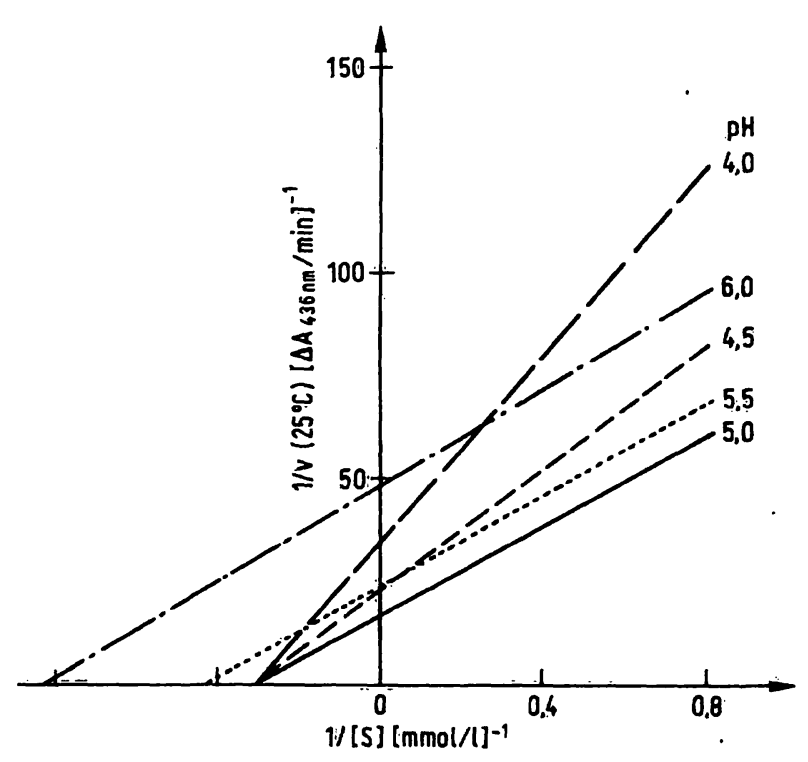

Abb. 7. Michaelis-Konstante der Peroxidase fü o-Phenylendiamin bei verschiedenen $\mathrm{pH}$-Werten. Aktivitätsbestimmung von $50 \mathrm{ng} / \mathbf{A}$ Peroxidase in 0,1 mol/1 Natriumcitrat; $5 \mathrm{mmol} / 1 \mathrm{H}_{2} \mathrm{O}_{2}$ und den entsprechenden Konzentrationen an 0 -Phenylendiamin bei den angegebenen pH-Werten. Reaktionstemperatur: $25{ }^{\circ} \mathrm{C}$. Darstellung nach Lineweaver-Burk. 
Tab. 1. Abhängigkeit der Peroxidaseaktivität vom eingesetzten o-Phenylenendiamin-Präparat.

In $0,1 \mathrm{~mol} / 1$ Natriumcitrat vom pH 5,0 wird die Aktivität von $100 \mathrm{ng} / 1$ Peroxidase in Anwesenheit von $5 \mathrm{mmol} / \mathrm{l}$ $\mathrm{H}_{2} \mathrm{O}_{2}$ und $15 \mathrm{mmol} / 1$ o-Phenylendiamin unterschiedlicher Präparate bei einer Raumtemperatur von $25^{\circ} \mathrm{C}$ photometrisch gemessen und die Absorptionsdifferenz pro 30 Minuten bei der Wellenlänge $436 \mathrm{~nm}$ angegeben.

\begin{tabular}{llr}
\hline Präparat des o-Phenylendiamin & $\begin{array}{l}\text { Peroxidase-Aktivität } \\
\left(\Delta \mathrm{A}_{30} \mathrm{~min} / 436 \mathrm{~nm}\right.\end{array}$ & (\%) \\
& $\left.\left(25^{\circ} \mathrm{C}\right)\right)$ & \\
\hline o-Phenylendiamin: Merck & 0,960 & 100 \\
o-Phenylendiamin: Fluka & 0,723 & 75 \\
o-Phenylendiamin/2 HCl: Fluka & 0,383 & 40 \\
o-Phenylendiamin/2 HCl: Sigma & 0,333 & 35 \\
\hline
\end{tabular}

während mit dem $o$-Phenylendiamin-dihydrochlorid generell tiefere Werte erreicht werden. Die Ursache für dieses unterschiedliche Verhalten des o-Phenylendiamin konnte noch nicht abgeklärt werden. Aufgrund dieser Resultate wurde für unsere Versuche stets das Substrat von Merck eingesetzt.

\section{Peroxidaseaktivität in Abhängigkeit zur Inkubations- temperatur}

Allgemein ist die katalytische Aktivität der Enzyme sehr stark von der Reaktionstemperatur abhängig. Eine Erhöhung der Temperatur um $1{ }^{\circ} \mathrm{C}$ bewirkt generell einen Anstieg der Reaktionsgeschwindigkeit um $5-15 \%$.

Die katalytische Reaktion der Peroxidase mit den Substraten $\mathrm{H}_{2} \mathrm{O}_{2}$ und $o$-Phenylendiamin unter den gegebenen Reaktionsbedingungen zeigt eine anders geartete Temperatürabhängigkeit. Während im Temperaturbereich von $18-26^{\circ} \mathrm{C}$ praktisch eine gleiche Peroxidaseaktivität gemessen wird, sinkt diese bei höheren Reaktionstemperaturen stark ab. Diese Resultate sind dadurch zu erklären, daß mit steigender Temperatur die Inaktivierung der Peroxidase durch das $\mathrm{H}_{2} \mathrm{O}_{2}$ schneller zunimmt als die katalytische Substratumsetzung des noch aktiven Enzyms.

Aufgrund dieser Resultate kann die Aktivitätsbestimmung der Peroxidase unter den gegebenen Testbèdingungen bei Raumtemperatur durchgefuihrt werden, ohne daß eine bestimmte Temperatur innerhalb von $18=26{ }^{\circ} \mathrm{C}$ eingehalten werden muß.

\section{Literatur}

1. Gallati, H. (1979) J. Clin. Chem. Clin. Biochem. 17, 1-7.

2. Omote, J., Nakada, J., Kobayashi, R. \& Sugiyama, N. (1971) Chem. Ind. 35, 996.

3. Maehly, A. C. (1955) Meth. Enzymol. 2, 801-813.

4. Yamazaki, I., Yokota, K. \& Nakajima, R. (1965) In:

'Oxidases Related Redox Systems' (King, T., Mason, H.

\& Morrison, M. eds.), New York, 485-504.

\section{Optimierte Bestimmungsmethode der Peroxidase}

Zur quantitativen Bestimmung der Peroxidase wird das Enzym in $1,0 \mathrm{ml}$ Substrat-Pufferlösung $(0,1 \mathrm{~mol} / 1$ Natriumcitrat vom pH $5,0 \mathrm{mit} 5 \mathrm{mmol} / 1 \mathrm{H}_{2} \mathrm{O}_{2}$ und $15 \mathrm{mmol} / 1 \mathrm{o}$-Phenylendiamin) während 30 Minuten bei Raumtemperatur $\left(18-26^{\circ} \mathrm{C}\right)$ lichtgeschützt inkubiert. Nach Ablauf der Reaktionszeit werden zum Abstoppen der katalytischen Aktivität $1,0 \mathrm{ml}$ Salzsäure $4 \mathrm{~mol} / 1$ zugemischt und die Farbintensität innerhalb von 60 Minuten bei der Wellenlänge $492 \mathrm{~nm}$ gemessen.

Die Abbildung 8 zeigt die lineare Proportionalität der Peroxidasekonzentration zur gemessenen Farbintensität nach einer enzymatischen Reaktion von 30 Minuten. Die Nachweisgrenze liegt bei $2 \mathrm{ng} / \mathrm{l}$. Der Meßbereich, der bei der Abbildung bis $100 \mathrm{ng} / 1$ Peroxidase reicht, kann erweitert werden, indem entweder die Peroxidase entsprechend vorverdünnt wird, oder die Dauer der Peroxidaseaktivität verkürzt und/oder das Volumen zum Abstoppen der katalytischen Reaktion vergrößert wird.

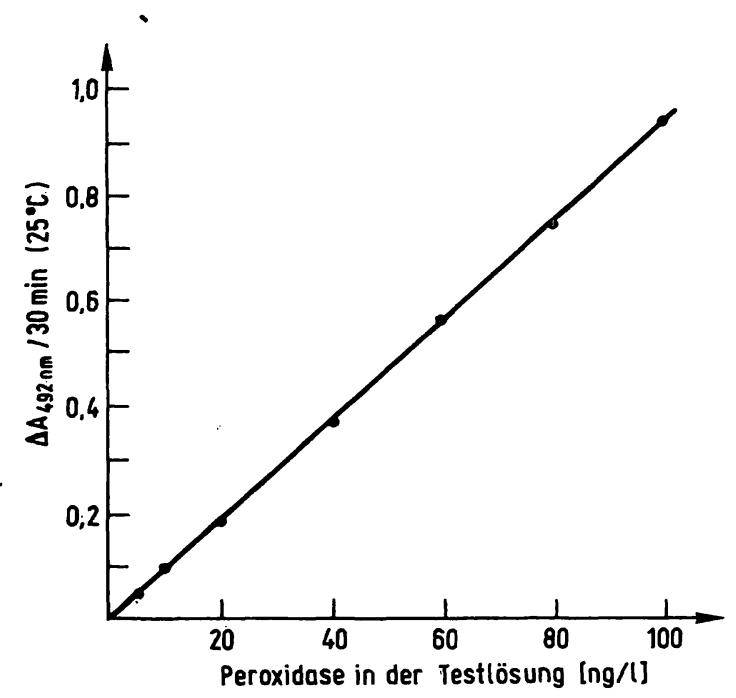

Abb. 8. Abhängigkeit der Absorptionsdifferenz von der Peroxidasekonzentration.

Die angegebenen Peroxidasemengen werden in $0,1 \mathrm{~mol} / 1$ Natriumcitrat vom pH 5,0 mit $5 \mathrm{mmol} / 1 \mathrm{H}_{2} \mathrm{O}_{2}$ und $15 \mathrm{mmol} / 1$ o-Phenylendiamin während 30 Minuten bei $25^{\circ} \mathrm{C}$ inkubiert. Anschließend wird die katalytische Reaktion durch Zugabe von $1,0 \mathrm{ml} 4 \mathrm{~mol} / 1$ Salzsäure pro $1,0 \mathrm{ml}$ Testlösung abgestoppt und die Farbintensität bei der Wellenlänge $492 \mathrm{~nm}$ gemessen.
5. Shindler, J., Childs, R. \& Bardsley, W. (1976) Eur. J. Biochem. $65,325-331$.

6. Dixon, M. (1953) Biochem. J. 55, 161-170.
Dr. H. Gallati

Diagnostische Forschungs- und

Entwicklungsabteilung

F. Hoffmann-La Roche \& Co. AG

Grenzacherstr. 124

CH-4002 Basel 
